University of Nebraska - Lincoln

DigitalCommons@University of Nebraska - Lincoln

\title{
All-Optical Steering Of Laser-Wakefield-Accelerated Electron Beams
}

A. Popp

Max-Planck-Institut für Quantenoptik

J. Vieira

GoLP/Istituto de Plasmas e Fusao Nuclear

J. Osterhoff

Max-Planck-Institut für Quantenoptik

Zs. Major

Max-Planck-Institut für Quantenoptik

R. Horlein

Max-Planck-Institut für Quantenoptik

See next page for additional authors

Follow this and additional works at: https://digitalcommons.unl.edu/physicsfuchs

Popp, A.; Vieira, J.; Osterhoff, J.; Major, Zs.; Horlein, R.; Fuchs, Matthias; Weingartner, R.; Rowlands-Ree, T.P.; Marti, M.; Fonseca, R.A.; Martins, S.F.; Silva, L.O.; Hooker, Simon M.; Krausz, F.; Gruner, F.; and Karsh, Stefan, "All-Optical Steering Of Laser-Wakefield-Accelerated Electron Beams" (2010). Matthias Fuchs Publications. 5.

https://digitalcommons.unl.edu/physicsfuchs/5

This Article is brought to you for free and open access by the Research Papers in Physics and Astronomy at DigitalCommons@University of Nebraska - Lincoln. It has been accepted for inclusion in Matthias Fuchs Publications by an authorized administrator of DigitalCommons@University of Nebraska - Lincoln. 


\section{Authors}

A. Popp, J. Vieira, J. Osterhoff, Zs. Major, R. Horlein, Matthias Fuchs, R. Weingartner, T.P. Rowlands-Ree, M. Marti, R.A. Fonseca, S.F. Martins, L.O. Silva, Simon M. Hooker, F. Krausz, F. Gruner, and Stefan Karsh 


\title{
All-Optical Steering of Laser-Wakefield-Accelerated Electron Beams
}

\author{
A. Popp, ${ }^{1,2}$ J. Vieira, ${ }^{3}$ J. Osterhoff, ${ }^{1,2}$ Zs. Major, ${ }^{1,2}$ R. Hörlein,,${ }^{1,2}$ M. Fuchs,,${ }^{1,2}$ R. Weingartner, ${ }^{1,2}$ T. P. Rowlands-Rees, ${ }^{4}$ \\ M. Marti, ${ }^{3}$ R. A. Fonseca, ${ }^{3}$ S. F. Martins, ${ }^{3}$ L. O. Silva, ${ }^{3}$ S. M. Hooker, ${ }^{4}$ F. Krausz, ${ }^{1,2}$ F. Grüner, ${ }^{1,2}$ and S. Karsch ${ }^{1,2, *}$ \\ ${ }^{1}$ Max-Planck-Institut für Quantenoptik, Hans-Kopfermann-Straße 1, 85748 Garching, Germany \\ ${ }^{2}$ Department für Physik, Ludwig-Maximilians-Universität München, Am Coulombwall 1, 85748 Garching, Germany \\ ${ }^{3}$ GoLP/Instituto de Plasmas e Fusão Nuclear, Instituto Superior Técnico, 1049-001 Lisboa, Portugal \\ ${ }^{4}$ Clarendon Laboratory, University of Oxford, OX1 3PU, United Kingdom \\ (Received 18 June 2009; revised manuscript received 23 July 2010; published 16 November 2010)
}

\begin{abstract}
We investigate the influence of a tilted laser-pulse-intensity front on laser-wakefield acceleration. Such asymmetric light pulses may be exploited to obtain control over the electron-bunch-pointing direction and in our case allowed for reproducible electron-beam steering in an all-optical way within an $8 \mathrm{mrad}$ opening window with respect to the initial laser axis. We also discovered evidence of collective electronbetatron oscillations due to off-axis electron injection into the wakefield induced by a pulse-front tilt. These findings are supported by 3D particle-in-cell simulations.
\end{abstract}

DOI: 10.1103/PhysRevLett.105.215001

PACS numbers: $52.38 . \mathrm{Kd}, 41.75 . \mathrm{Jv}$

Over the past decades, the concept of laser-wakefield acceleration (LWFA) of electrons has evolved from an idea [1] into a technology ready for first applications. The development of high-power ultra-short-pulse lasers [2] provided the means for the first experimental generation of laser-driven quasimonoenergetic electron bunches [3-5] that now can reach up to $\mathrm{GeV}$ energies [6]. For a reliable use of these electrons, e.g., in compact soft x-ray undulatorradiation sources [7] or eventually in free-electron lasers [8], control over the LWFA mechanism and thus over electron-bunch parameters is of crucial importance. This requires a profound understanding of the underlying processes, including the impact of plasma and laser-pulse characteristics. In LWFA a high-intensity laser pulse drives a strong plasma wave that features longitudinal electric fields exceeding $10 \mathrm{GV} / \mathrm{m}$. Electrons of sufficient momentum may be trapped inside this wake and can be efficiently accelerated to ultrarelativistic energies. As external injection of electrons into the wakefield poses several technical challenges, most LWF accelerators rely on self-trapping, which may occur if the plasma wave is excited in a nonlinear wave-breaking regime. This so-called bubble regime may be accessed when the driving laser-pulse length is comparable to half the plasma wavelength with a normalized vector potential $a_{0}>1$. These pulse conditions are usually only met after substantial nonlinear self-modulation inside the plasma. The nonlinearity of such processes puts high demands on the shot-to-shot stability of the laser and gas target properties, which when controlled well, allow for the production of reproducible electron beams [9]. This Letter reports on the influence of an often neglected laser parameter, the pulse-front tilt (PFT), on LWFA. For a temporally compressed pulse, PFT is equivalent to an angular chirp (AC) in the laser near-field as will be discussed in the following. Usually, high-intensity lasers are based on the chirped-pulse-amplification principle [2], in which the laser pulses are first stretched in time, then amplified and subsequently temporally compressed. Often a grating stretcher-compressor setup is used. A slight misalignment of such a grating compressor [cf. Fig. 1(a)] will result in AC leading to a pulse intensity envelope that is tilted with respect to the laser-propagation direction $z$. The electric field of an angularly chirped pulse can be described by $\tilde{E}\left(k_{x}, k_{z}, \omega\right)=\tilde{E}_{k_{x}, k_{z}}\left(k_{x}-g \omega\right) \tilde{E}_{\omega}(\omega)$ in the $k-\omega$ domain ( $\omega$ : laser frequency, $k_{x, z}$ : spatial wave vector). Here, the pulse is angularly chirped in the $x-z$ plane. The parameter $g$ determines the angular offset between different frequencies and can be connected to the groove spacing $s$, the diffraction order $m$, and the diffraction angle $\beta_{0}$ of a single grating [cf. Fig. 1(a)] by $g=m \lambda_{0}^{2} /\left(\operatorname{cs} \cos \beta_{0}\right) . \quad \lambda_{0}$ denotes the central laser wavelength and $c$ the speed of light in vacuum. Applying a Fourier transform into the $x$ - $t$ domain yields $E(x, z, t)=E_{x, z}(x, z) E(t-g x)$ representing a tilted laserpulse intensity front. The angular dispersion $d \varphi$ per wavelength $d \lambda$ is then given by $d \varphi / d \lambda=c g / \lambda_{0}$ [10] and the PFT angle $\psi$ by $\tan \psi=\lambda_{0} d \varphi / d \lambda$. The presence of AC in the near field of an ultrashort pulse has important implications on the properties of the far-field. As different laser wavelengths are incident onto a focusing optic under different angles, the AC causes a spatial chirp in the focal plane. This leads to a twofold reduction of intensity: the transverse size of the focal spot increases in the chirp direction and the presence of a spatial chirp results in a locally reduced spectral bandwidth and thus in an increased pulse duration. Consequently, the laser pulse exhibits no PFT at the focal plane. As can be seen in Fig. 1(b), when focusing a tilted laser pulse, the tilt angle first increases when the beam approaches the focus and the transverse beam width decreases. However, within the Rayleigh length $l_{R}$ the AC causes a spatial chirp and the intensity-front tilt vanishes. As the pulse propagates through focus the PFT flips in the plane of $\mathrm{AC}$ and further evolves in a point-symmetric fashion. In 


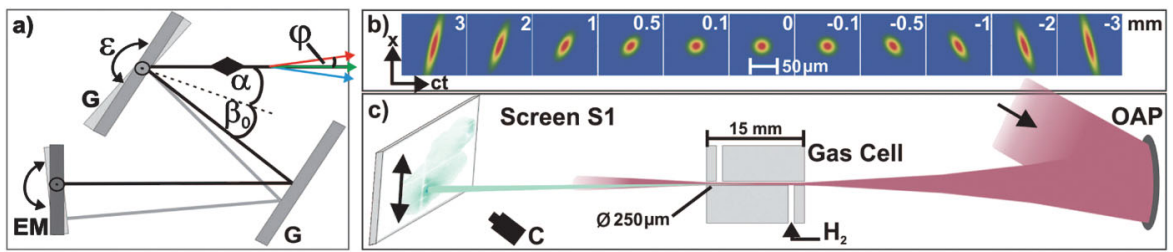

FIG. 1 (color). (a) Grating-compressor setup. (b) Calculated PFT evolution for the ATLAS laser pulse far-field with $d \varphi / d \lambda=$ $38 \mu \mathrm{rad} / \mathrm{nm}$ (focused by an $f=1.50 \mathrm{~m}$ OAP to $22 \mu \mathrm{m}$ FWHM diameter). (c) Electron acceleration setup. The angles $\epsilon, \varphi, \alpha, \beta_{0}, \psi$ are explained in the text. ( $\mathrm{G}$ denotes grating, EM end mirror, OAP off-axis parabola, $\mathrm{C}$ CCD camera).

order to better understand the implications of driving a wakefield with a pulse-front-tilted beam, we conducted LWFA experiments while scanning the laser AC by detuning the relative angle between the compressor gratings. The results show that PFT directly influences the pointing direction of an accelerated electron bunch. Its deviation angle from the initial laser direction increases with increasing tilt. This can be used to steer the quasimonoenergetic part of the electron bunch with no significant chromatic aberrations. Furthermore, we have observed evidence of collective electron-betatron oscillations induced by PFT. Our findings were validated by 3D particle-in-cell (PIC) simulations. Figure 1 shows a schematic view of the experimental setup. The ATLAS Ti:sapphire-laser system at the MPI für Quantenoptik was used as the LWFA-driver, delivering $850 \mathrm{~mJ}$ pulses of $\tau_{0}=37 \mathrm{fs}$ FWHM duration on target at $\lambda_{0}=800 \mathrm{~nm}$. These pulses were focused by an $f / 22$ offaxis parabola to a spot size $d$ of $23 \mu \mathrm{m}$ FWHM diameter $\left(a_{0}=0.95\right.$ for zero AC with a Strehl ratio of $\left.>0.7\right)$ into a $15 \mathrm{~mm}$-long steady-state-flow gas cell (electron density $\left.n_{e}=7.7 \times 10^{18} \mathrm{~cm}^{-3}\right)$. This setup is characterized in more detail in [9]. Owing to the stable interaction environment and small shot-to-shot variations in laser energy of $\sim 2 \% \mathrm{rms}$, the accelerated electron bunches exhibited good stability in all monitored beam parameters, the most important of which was the excellent pointing stability of $1.7 \mathrm{mrad} \mathrm{rms}$. This reproducibility in combination with an electron-injection rate of close to $100 \%$ allowed for the observation of small deviations in the pointing direction with meaningful statistics. The beam pointing was measured by imaging a scintillating screen S1 situated $1.12 \mathrm{~m}$ behind the gas-cell exit onto a 12-bit CCD camera [Fig. 1(b)]. S1 could be removed to allow the electron bunch to propagate unscattered through a dipole field of $\sim 0.5 \mathrm{~T}$, in order to detect the electron-energy spectra on a second scintillating screen $\mathrm{S} 2$ behind the spectrometer magnet. The spectra typically displayed a broad electron background with a quasimonoenergetic feature and a high-energy cutoff at $170 \pm 7 \mathrm{MeV}$ rms independent of PFT, similar to the results shown in [9]. However, for the largest measured PFT only those shots of the pointing distribution with lower angular deviations could be evaluated given the limited acceptance angle of the spectrometer. The accelerated charge above $100 \mathrm{MeV}$ is $23 \pm 6 \mathrm{pC}$ rms as deduced from $\mathrm{S} 2$, which was cross referenced with calibrated image plates [12]. The AC was introduced by rotating one compressor grating around the axis parallel to its grooves [Fig. 1(a)]. After the return pass through the compressor the $\mathrm{AC} d \varphi / d \lambda$ is then related to the relative grating rotation angle $\epsilon$ by $d \varphi / d \lambda=2 \epsilon \tan \beta_{0} /(s \cos \alpha)$ [13]. Here, $\alpha$ is the angle of incidence. In order to impose ACs of up to $1.5 \mu \mathrm{rad} / \mathrm{nm}$ the grating had to be detuned by a maximum of $0.17 \mathrm{mrad}$. Subsequently, the beam path was realigned by turning the compressor end mirror. A DAZZLER [14] was used to recompress the pulses to their shortest possible duration. The absolute value of PFT in the laser near-field was measured using a spectrally resolved inverted field interferometer [15]. This method is insensitive to a temporal chirp in the laser pulse. Its inherent measurement error can be estimated to not exceed $\pm 0.1 \mu \mathrm{rad} / \mathrm{nm}$.

Figures 2(a) and 2(b) display the electron pointing for different amounts of AC. Clearly, the center of mass of the distribution shifts along the $x$ axis as a function of AC. In all measurements electron injection occurred at every shot for a background electron density of $7.7 \times 10^{18} \mathrm{~cm}^{-3}$. Only for the largest $\mathrm{AC}$ of $\sim 1.6 \mu \mathrm{rad} / \mathrm{nm}$ the density had to be increased to $9.9 \times 10^{18} \mathrm{~cm}^{-3}$ to ensure a high trapping probability despite the reduction of laser intensity at focus due to the high AC. For an AC of $2.3 \mu \mathrm{rad} / \mathrm{nm}$ (corresponding to a reduction of $a_{0}$ by a factor of 1.8) and above no injection could be achieved. In $\sim 50 \%$ of all shots with $\mathrm{AC} \geq 1 \mu \mathrm{rad} / \mathrm{nm}$ two or more electron bunches were recorded simultaneously on the screen. Those shots were omitted from the analysis and are not shown in Fig. 2. In addition, the pointing fluctuations increased from $1.7 \mathrm{mrad}$ $\mathrm{rms}$ at no $\mathrm{AC}$ to $4.8 \mathrm{mrad} \mathrm{rms}$ for the highest $\mathrm{AC}$. The reduced stability can partially be attributed to the less reliable injection process for higher PFTs, i.e., lower laser intensities. In addition, the simulations described below show that the presence of a PFT in the driver pulse facilitates off-axis electron injection into the wakefield. The betatron oscillations induced in this way may also deteriorate the electron-pulse quality. However, already small ACs $(\sim 1 \mu \mathrm{rad} / \mathrm{nm})$ result in a deflection of the electron beam from the optical axis of up to $\pm 4 \mathrm{mrad}$ while the pointing fluctuations remain still small $(<2.5 \mathrm{mrad} \mathrm{rms})$. This effect can therefore be exploited to steer the electron bunches. These findings show that the influence of the laser PFT on LWFA is significant and has been underestimated to date. We note that for our focusing conditions an $\mathrm{AC}$ of $1 \mu \mathrm{rad} / \mathrm{nm}$, causing a $4 \mathrm{mrad}$ deflection of the electron beam, leads to an elliptical laser focus with only a factor of 


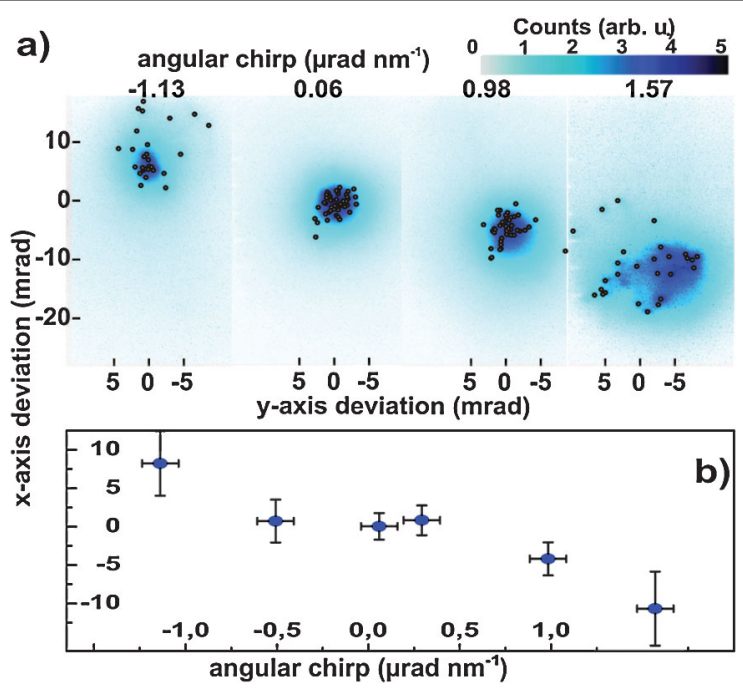

FIG. 2 (color). (a) Individual peak positions and the summed signal of electron beams on screen S1. Each series of shots was generated with a different AC. (b) Deviation of the electron bunches from the laser axis versus AC. The dots represent the center of mass of the electron-beam-pointing distributions as shown in (a). The vertical error bars result from the rms electronpointing fluctuations, the horizontal ones from the error of the $\mathrm{AC}$ measurement. For the highest AC, injection only occurred for an increased electron density.

1.15 between the major and minor axes. Therefore, it is insufficient to extract information about the residual AC merely by the shape of the focal spot. This parameter must be monitored carefully, especially if alignment sensitive devices such as undulators need to be positioned relative to the electron-beam trajectory.

We employed the full 3D PIC code OSIRIS [11] to gain insight into the LWFA process when driven by a tilted laser pulse. For the simulation results shown in Figs. 3 (first row) and 4 the laser pulse was initialized at focus without PFT, but with spatial chirp and an accordingly increased pulse width and length and hence a decreased pulse intensity. The spatial chirp $d x / d \lambda$ at focus and the AC in the collimated beam are connected through $d \varphi / d \lambda=(d x / d \lambda) / f$, with $f$ being the focal length of the focusing optic. The pulse propagates in the $z$ direction, is linearly polarized along $y$, and the spatial and angular chirps are oriented along the $x$ axis. We used a Gaussian transverse profile and a symmetric polynomial temporal profile, with $\lambda_{0}=$ $800 \mathrm{~nm}$. The parameters of the full-scale 3D simulations shown in Fig. 3 closely resemble the experiment: $\tau_{0}=$ $37 \mathrm{fs}, d=22 \mu \mathrm{m}, a_{0}=1$. In the case where an AC was present, these values were adapted accordingly, while pulse energy and spectral content were kept constant. The preformed plasma has a uniform electron density of $7.7 \times 10^{18} \mathrm{~cm}^{-3}$, and the ions form a neutralizing immobile background. The simulation box, which moves at $c$, is $70 \times 70 \times 30 \mu \mathrm{m}^{3}$, and is divided into $350 \times 350 \times 2000$ cells for the transverse and longitudinal directions, respectively, with 2 particles per cell.

In Fig. 3 it is evident that as soon as the PFT develops, the laser pulse is deflected away from the initial laser axis. This can be attributed to the transverse plasma-density gradient caused by the tilted pulse front, which corresponds to a varying refractive index perpendicular to the laser-propagation direction. The transverse motion of the laser pulse due to the PFT increases the offset between the bubble and the self-injected beam. As a result, the transverse electric field pushes the electron beam towards the bubble center. Thus, the average perpendicular velocity of the electron beam approaches the transverse bubble velocity and the electron beam leaves the plasma under an angle that is mainly determined by the wakefield direction, as indicated by the experimental results.

In addition to the angular deviation of the electron bunch measured on S1 another effect was observed both experimentally and in simulation. On S2 the electron bunches are spectrally resolved in one dimension, whereas the perpendicular axis gives spatial information, which in our case corresponds to the plane in which the symmetry is broken by PFT. Some of the electron beams generated from tiltedpulse driven LWFA leave a corrugated trace on this screen [see Fig. 4(a)], corresponding to a variation of beam pointing with electron energy. This wiggled electron trace may

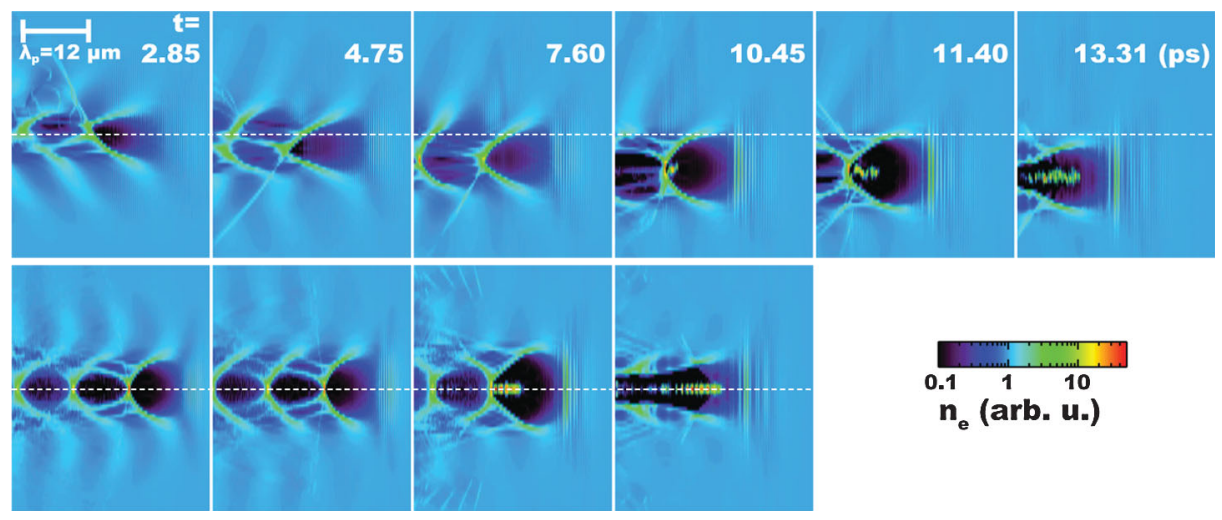

FIG. 3 (color). Electron density in the central $x$ - $z$ plane of a 3D LWFA-simulation volume with (top) and without (bottom) a pulsefront-tilted driver laser $\left(\tau_{0}=37 \mathrm{fs}, d=22 \mu \mathrm{m}, a_{0}=1.0, n_{e}=7.7 \times 10^{18} \mathrm{~cm}^{-3}, d \varphi / d \lambda=1 \mu \mathrm{rad} / \mathrm{nm}\right)$. 


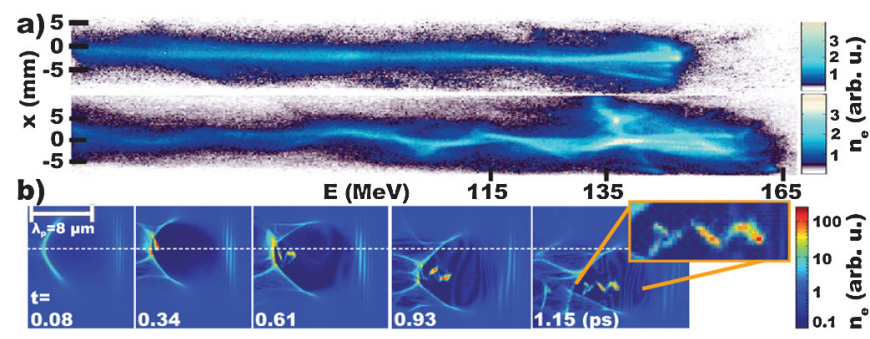

FIG. 4 (color). (a) Measured electron spectra on S2, top: no PFT in the driver laser, bottom: PFT present. (b) 3D simulation of extreme case $\left(\tau_{0}=17 \mathrm{fs}, \quad d=5 \mu \mathrm{m}, a_{0}=3, n_{e}=\right.$ $17 \times 10^{18} \mathrm{~cm}^{-3}, \quad d \varphi / d \lambda=0.008 \mu \mathrm{rad} / \mathrm{nm}$ assuming $f=$ $50 \mathrm{~cm}$ ) showing off-axis injection of electrons into the wakefield and collective electron-betatron oscillations.

be experimental evidence of collective betatron oscillations performed by the electrons trapped in the wake. Glinec et al. [16] have explained similar observations by the presence of higher-order asymmetric modes of the driving laser. Mangles et al. [17] created a wave front with coma to achieve similar results. Our experiments, however, suggest that the observed oscillations can also be induced by a PFT in the laser beam. In our configuration, these oscillations are a direct result of off-axis injection of electrons due to asymmetric plasma wake fields driven by a tilted laser pulse. In this case all electrons trapped at a similar localized off-axis position on a time scale $\Delta t$ shorter than the characteristic evolution time of the PFT $\left(\Delta t \ll l_{R} / c\right)$ acquire about the same transverse momentum when oscillating in the transverse, focusing electric field of the wake. Hence, they can perform collective betatron motions [see Fig. 4(b)]. In the case of a driver pulse without PFT the plasma wave and thus the injection region is cylindrically symmetric.

For a quantitative comparison between experiment and simulation, the simulated mean angular deviation $\phi_{s}$ of the self-injected electron bunch was extracted from its transverse momentum in the direction of the PFT. As long as the transverse focusing fields of the wakefield are present, $\phi_{s}$ is mainly determined by the betatron motion of the electron bunch within the bubble and oscillates from -5 to $20 \mathrm{mrad}$. The fact that $\phi_{s}$ oscillates at all reflects the broken symmetry during injection and the in-phase oscillation of electrons in the bunch. The fact that $\phi_{s}$ does not oscillate symmetrically around zero can be attributed to the shifting wakefield structure. As soon as the laser starts to deplete, these oscillations are damped to 1-3 mrad. At the very end of the gas cell, when in our case the laser is entirely depleted and the wakefield is no longer driven, the mean angular deviation of the electron bunch stabilizes at $1.4 \mathrm{mrad}$, consistent with the deviation of the bubble. Shortly before laser energy depletion becomes significant and the bubble structure starts dissolving, the bubble has an angular offset of $2.6 \mathrm{mrad}(d \varphi / d \lambda=1 \mu \mathrm{rad} / \mathrm{nm}$, assuming a focal length of $1.54 \mathrm{~m}$ ). The final net deviation of
$1.4 \mathrm{mrad}$ of the electron bunch, after the betatron oscillations were damped during the propagation through the residual plasma, is associated with the transverse deviation of the blowout region and is in reasonable agreement with the experimental results $(4.2 \mathrm{mrad}$ for $d \varphi / d \lambda=$ $0.98 \mu \mathrm{rad} / \mathrm{nm}$ ).

In summary, we have demonstrated the influence of a PFT on LWFA. Such a tilt may be used to excite asymmetric plasma wakes, which can steer electron bunches away from the initial laser axis. This also implies that the PFT needs to be carefully monitored if one wants to avoid this effect. Moreover, the excitation of collective electron-betatron oscillations by PFT seems feasible. This is potentially interesting as the collectively oscillating electrons emit radiation, which may be coherent [18] and in the keV-energy range (cf. e.g., [19]). Furthermore a potential knob to tune the radiation wavelength is introduced, as the strength of PFT changes the off-axis distances for injection (see also [17]). However, further experimental investigations are necessary to establish how exactly the PFT changes the properties of betatron radiation.

This work was funded by TR18, MAP (DFG), and EURATOM-IPP. The work of J. V., S.F.M., M. M., R.A.F., and L.O.S. is partially supported by FCT (Portugal).

*Corresponding author. stefan.karsch@mpq.mpg.de

[1] T. Tajima and J. M. Dawson, Phys. Rev. Lett. 43, 267 (1979).

[2] D. Strickland and G. Mourou, Opt. Commun. 55, 447 (1985).

[3] J. Faure et al., Nature (London) 431, 541 (2004).

[4] S.P.D. Mangles et al., Nature (London) 431, 535 (2004).

[5] C. G. R. Geddes et al., Nature (London) 431, 538 (2004).

[6] W. P. Leemans et al., Nature Phys. 2, 696 (2006).

[7] M. Fuchs et al., Nature Phys. 5, 826 (2009).

[8] F. Grüner et al., Appl. Phys. B 86, 431 (2007).

[9] J. Osterhoff et al., Phys. Rev. Lett. 101, 085002 (2008).

[10] S. Akturk et al., Opt. Express 12, 4399 (2004).

[11] R. Fonseca et al., Lect. Notes Comput. Sci. 2331, 342 (2002).

[12] K. A. Tanaka et al., Rev. Sci. Instrum. 76, 013507 (2005).

[13] G. Pretzler et al., Appl. Phys. B 70, 1 (2000).

[14] P. Tournois, Opt. Commun. 140, 245 (1997).

[15] K. Varjú et al., Appl. Phys. B 74, s259 (2002).

[16] Y. Glinec et al., Europhys. Lett. 81, 64001 (2008).

[17] S.P.D. Mangles et al., Appl. Phys. Lett. 95, 181106 (2009).

[18] F. Albert et al., Plasma Phys. Controlled Fusion 50, 124008 (2008).

[19] E. Esarey et al., Phys. Rev. E 65, 056505 (2002). 